\title{
Heavy Elements and Cool Stars
}

\author{
Glenn M. Wahlgren ${ }^{*, \dagger}$ Kenneth G. Carpenter* and Ryan P. Norris ${ }^{*, \dagger}$ \\ *NASA-GSFC, Code 667, Greenbelt, MD, USA 20071 \\ ${ }^{\dagger}$ Catholic Univ of America, Dept of Physics, 620 Michigan Ave NE, Washington DC, USA 20064
}

\begin{abstract}
We report on progress in the analysis of high-resolution near-IR spectra of $\alpha$ Orionis (M2 Iab) and other cool, luminous stars. Using synthetic spectrum techniques, we search for atomic absorption lines in the stellar spectra and evaluate the available line parameter data for use in our abundance analyses. Our study concentrates on the post iron-group elements copper through zirconium as a means of investigating the slow neutron-capture process of nucleosynthesis in massive stars and the mechanisms that transport recently processed material up into the photospheric region. We discuss problems with the atomic data and model atmospheres that need to be addressed before theoretically derived elemental abundances from pre-supernova nucleosynthesis calculations can be tested by comparison with abundances determined from observations of cool, massive stars.
\end{abstract}

Keywords: atomic data, stars: abundances, stars: supergiants, stars: individual ( $\alpha$ Orionis)

PACS: $95.30 . \mathrm{Ky}$, 95.75.Fg, 97.10.Cv, 97.10.Ex, 97.10.Tk, 97.20.Pm

\section{ELEMENTAL ABUNDANCES AND THE WEAK S-PROCESS}

Nucleosynthesis by the slow neutron capture process ( $s$-process) is associated with different stellar sites. The main $s$-process is active in the interior regions of asymptotic giant-branch stars and contributes to the creation of all elements heavier than zinc up to and including bismuth. A variant of the $s$-process is the weak s-process, which is attributed to a lower neutron exposure and occurs in massive supergiants during the phases of He-core burning and $\mathrm{C}$-shell burning. Isotopes in the atomic mass range $A$ $=70$ to 100 play a role in the weak $s$-process. Calculations (c.f. [1]) utilizing large nuclear reaction networks show that $s$-process efficiency increases with stellar mass in the range 15 to 30 solar masses and predict overproduction factors for the $s$-process nucleosynthesis to be highest for certain isotopes of $\mathrm{Ge}, \mathrm{Se}, \mathrm{Kr}, \mathrm{Sr}$, and $\mathrm{Y}$.

The analysis of post iron-group element abundances for massive supergiants has been limited. Studies by [2-6] determined element abundances from optical region spectra for $\mathrm{G}$ and $\mathrm{K}$ type Ib supergiants. For these low-mass supergiants no abundance patterns were identified that could be interpreted as the dredge-up of recently processed material. The infrared (IR) spectrum $(1.11-1.35 \mu \mathrm{m})$ of $\alpha$ Orionis (M2 Iab) was investigated by [7] for elements no heavier than zinc, finding most abundances to be metal deficient.

We are undertaking the analysis of spectra of cool, luminous stars. Our goals are to identify spectral lines at IR wavelengths for elements heavier than iron and to determine abundances for heavy elements in massive supergiants for comparison with theoretical predictions for abundances produced by the weak $s$-process. Here, we report on the availability of the atomic data required for this analysis and we present preliminary abundance results for the massive supergiant $\alpha$ Orionis. 


\section{ANALYZING THE SPECTRUM OF $\alpha$ ORIONIS}

This analysis makes use of high-resolution spectra obtained with the Nordic Optical Telescope SOFIN echelle spectrograph [8] $(\lambda=3600-11000 \AA, \mathrm{R}=25000, \mathrm{~S} / \mathrm{N}$ $=400)$, the VLT/UVES $(\lambda=3100-10400 \AA, \mathrm{R}=100000)$, and the KPNO 4m IR FTS $(\lambda=2.02-2.40 \mu \mathrm{m}, \mathrm{R}=91000,174000$ [9] and 3.6 $-4.1 \mu \mathrm{m}$ (Hinkle, private communication)). Spectral line identification and element abundances were determined by fitting the observed spectra with spectra generated using the SYNTHE [10] code, which employed a model atmosphere generated using the LTE, plane-parallel modelling code ATLAS12 [11].The atmospheric parameters $\left(T_{\text {eff }}=3550 \mathrm{~K}, \log g=-0.2, \mathrm{v}_{\text {micro }}\right.$ $=3.1 \mathrm{~km} \mathrm{~s}^{-1}, \mathrm{v}_{\text {macro }}=11 \pm 2 \mathrm{~km} \mathrm{~s}^{-1}$ ) were determined by fitting IR spectral features [12-13]. Molecules are accounted for in both the statistical equilibrium, line opacity and emergent spectrum. The assumption that supergiant atmospheres can be represented by plane-parallel LTE models is problematic. For example, our LTE abundance from the strong lines of the $4 d^{2} \mathrm{D}-5 p^{2} \mathrm{P}^{\mathrm{o}} \mathrm{Sr}$ II transition array near $1 \mu \mathrm{m}$ was reduced by 0.6 dex to correct for non-LTE modelling (Mashonkina 2006, private communication). Spectral lines from other elements may also require non-LTE line formation. Also, due to the great physical extent of the atmosphere, the need for spherical models in the synthetic spectrum calculations should be assessed.

The element abundances were determined from spectral lines at red and near-IR wavelengths (Wahlgren et al., in preparation), since for $\mathrm{M}$ type stars such as $\alpha$ Orionis the optical region is blanketed by absorption from the TiO molecule. We determine solar-like abundances for iron-group elements ( $\mathrm{Sc}, \mathrm{Ti}, \mathrm{V}, \mathrm{Cr}, \mathrm{Mn}, \mathrm{Fe}, \mathrm{Co}, \mathrm{Ni}$ ). To within uncertainties as large as 0.6 dex in some cases, post-iron elements $(\mathrm{Cu}, \mathrm{Rb}, \mathrm{Y}, \mathrm{Zr}$, $\mathrm{Nb}, \mathrm{Nd}, \mathrm{Hf})$ also display solar-like abundances, with the exception of $\mathrm{Ge}(+0.33 \mathrm{dex})$ and $\mathrm{Sr}(+1.4$ dex, non-LTE corrected). Enhancements of $\mathrm{Ge}$ and $\mathrm{Sr}$ are predicted from nucleosynthesis calculations for $s$-processing in massive stars. These enhancements may signal the dredge-up of processed material into the photosphere of $\alpha$ Orionis.

The availability of the free neutrons necessary for slow neutron-capture nucleosynthesis at interior layers can be inferred from determination of the surface ${ }^{12} \mathrm{C} /{ }^{13} \mathrm{C}$ ratio. For the solar system this ratio has a value of approximately 90 , while for $\alpha$ Orionis it has been determined to be 7 [14-16] from near-IR CN lines, 6 from IR CO lines [17], and between 10 and 20 [18] from UV CO lines. Harper et al. (this conference) present evidence for multiple layers of circumstellar material, which may impact these interpretations. Enhanced levels of ${ }^{13} \mathrm{C}$, brought to photospheric layers by convective processes, may signal the presence of free neutrons through the ${ }^{13} \mathrm{C}(\alpha, n){ }^{16} \mathrm{O}$ reaction.

\section{PROBLEMS WITH ATOMIC DATA}

Atomic lines are evident across the IR spectrum of $\alpha$ Orionis. Figure 1 shows that although spectral lines of atomic species are present at IR wavelengths, they may not be useful for the determination of element abundances due to poor quality atomic data, particularly the oscillator strength, or $f$-value. At this time experimental $f$-values exist for relatively few atomic lines at wavelengths longer than $1 \mu \mathrm{m}$. For the vast majority of IR atomic transitions their $f$-values have been determined from ab initio 


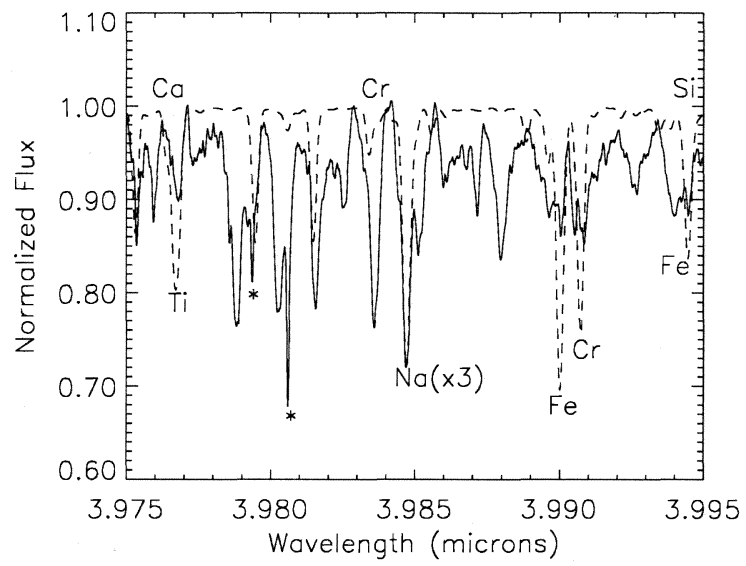

FIGURE 1. Atomic lines near $4 \mu \mathrm{m}$ in the KPNO FTS spectrum of $\alpha$ Orionis. Lines of the OH molecule in the synthetic spectrum (dashed) are unlabeled. Strong terrestrial lines are denoted by a star.

calculations, which have unquantified uncertainty. Astrophysical $g f$-values, typically determined from the solar spectrum, are another data source for IR transitions [19], but they should be used with caution when applied to the analysis of stars of a different spectral type.

For elements we associate with the weak $s$-process $(Z=30-42)$, there exist spectral lines from neutral and singly-ionized species at IR wavelengths, although they may not be visible in the cool star spectra that we analyse. The usefulness to abundance studies of these lines is dependent on the transition energy levels, plasma temperature and pressure, element abundance, and line blending. For lines at IR wavelengths, $g f$-values essentially do not exist for certain elements ( $\mathrm{Ge}, \mathrm{As}, \mathrm{Se}, \mathrm{Br}, \mathrm{Kr}, \mathrm{Zr}, \mathrm{Nb}, \mathrm{Mo}$ ) or are of high uncertainty (Zn, Ga, Rb, Sr, Y). The databases that astronomers typically access for atomic data, i.e. the lists of R. Kurucz [20], the Vienna Atomic Line Database [21] and the National Institute of Standards and Technology, are incomplete. For some species, the atomic data components necessary for constructing oscillator strengths (i.e. energy level lifetimes, transition probabilities, line intensities) exist in the research literature, but have not been reformulated into $g f$-values. In general, it can be said that abundance determinations for elements heavier than the iron group, using lines at IR wavelengths, require improvements to the current state of knowledge regarding oscillator strengths.

The IR spectrum analysis of $\alpha$ Orionis benefits from comparison with other cool stars, to strengthen line identications, understand line blending and establish element abundance trends. We analyse atomic features for $s$-process elements in spectra extracted from the ESO archives [22] and the $2.0-2.4 \mu \mathrm{m}$ FTS data [9]. Figure 2 compares the FTS spectra of stars of different chemical composition (carbon star TX Piscium, oxygen-rich star $\alpha$ Orionis, and S-type star $\chi$ Cygni). Other well-fit spectral features are molecular $(\mathrm{CN})$. The poor fit to the Y I line is the result of an incorrect $g f$-value, and the line is clearly present in the $\mathrm{C}$ and $\mathrm{S}$ stars. 


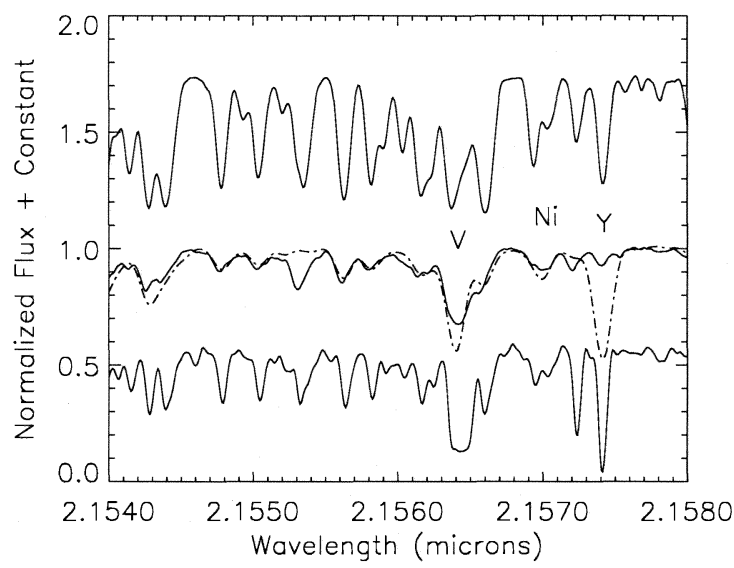

FIGURE 2. KPNO 4m FTS spectra (solid) for the stars TX Piscium (C6.2), $\alpha$ Orionis (M2 Iab), and $\chi$ Cyg (S7.1). A theoretical spectrum for $\alpha$ Orionis (dot-dash) shows good fits to many atomic and molecular features.

\section{ACKNOWLEDGMENTS}

GMW would like to acknowledge funding from NASA grant NNG06GJ29G.

\section{REFERENCES}

1. L.-S. The, M. F. El Eid and B. S. Meyers, ApJ 533, 9xx-1015 (2000).

2. G. A. Bakos JRASC 65, 222-238 (1971).

3. J. van Paradijs, $A \& A$ 23, 369-379 (1973).

4. R. E. Luck, ApJ 212, 743-753 (1977).

5. R. E. Luck and H. E. Bond, ApJ 241, 218-228 (1980).

6. R. E. Luck and H. E. Bond, ApJS 71, 559-581 (1989).

7. T. Vieira, Uppsala Astron. Obs. Report No. 32 (1986).

8. I. Ilyin, PhD Thesis, University of Oulu, Finland (2000).

9. L. Wallace and K. Hinkle, ApJS 107, 312-390 (1996).

10. R. L. Kurucz, Kurucz CD-ROM No. 18, SAO, Cambridge, Mass. (1993).

11. R. L. Kurucz, Mem. Soc. Astr. Italiana Suppl. 8, 10-20 (2005).

12. M. Lundqvist and G. M. Wahlgren, Nucl. Phys. A 358, 304 (2005).

13. M. Lundqvist, PhD Thesis, Lund Observatory, Lund University, Sweden (2006).

14. D. L. Lambert, D. S. Dearborn and C. Sneden, ApJ 193, 621-630 (1974).

15. K. H. Hinkle, D. L. Lambert and R. L. Snell, ApJ 210, 684-693 (1976).

16. M. J. Harris and D. L. Lambert, ApJ 281, 739-745 (1984).

17. A. P. Bernat, D. N. B. Hall, K. H. Hinkle and S. J. Ridgway, ApJ 233, L135-L138 (1979).

18. G. M. Wahlgren, R. D. Robinson and K. G. Carpenter, in Cool Stars, Stellar Systems and the Sun, eds M. S. Giampapa and J. A. Bookbinder, ASP Conf Ser 26, ASP, San Francisco 1992, pp.37-39.

19. J. Meléndez and B. Barbuy, ApJS 124, 527-546 (1999).

20. R. L. Kurucz and B. Bell, Kurucz CD-ROM No. 23, SAO, Cambridge, Mass. (1995).

21. F. Kupka, et al. A\&AS 138, 119-133 (1999).

22. S. Bagnulo et al., Library of High Resolution Spectra of Stars Across the H-R Diagram, www.sc.eso.org 